\title{
Using Neurofeedback to Lower PTSD Symptoms
}

\author{
Devon E. Romero ${ }^{1 *}$, Aneesa Anderson ${ }^{1}$, J. Claire Gregory ${ }^{1}$, Courtney A. Potts ${ }^{2}$, Ashley \\ Jackson', James R. Spears ${ }^{1}$, Mark S. Jones ${ }^{1}$, and Stacy Speedlin' \\ ${ }^{1}$ University of Texas at San Antonio, Department of Counseling, San Antonio, Texas, USA \\ ${ }^{2}$ University of Alabama, College of Education, Tuscaloosa, Alabama, USA
}

\begin{abstract}
This study examines the effectiveness of neurofeedback training for individuals presenting with a primary concern of posttraumatic stress disorder symptoms. The present study includes 21 adult clients with $62 \%(n=13)$ selfreporting as female. Participants completed pre- and postassessments including the Davidson Trauma Scale and Inventory of Altered Self-Capacities and participated in neurofeedback training sessions twice a week for one academic semester. Neurofeedback training involved decreasing 2-6 Hz and 22-36 Hz while increasing $10-13 \mathrm{~Hz}$ with a placement of T4 as the active site and P4 as the reference site. Study findings demonstrated statistically significant improvement in affect regulation and trauma symptom severity and frequency. We present limitations and implications for future research.
\end{abstract}

Keywords: PTSD; neurofeedback; trauma; affect regulation

Citation: Romero, D. E., Anderson, A., Gregory, J. C., Potts, C. A., Jackson, A., Spears, J. R., ... Speedlin, S. (2020). Using neurofeedback to lower PTSD symptoms. NeuroRegulation, 7(3), 99-106. https://doi.org/10.15540/nr.7.3.99

${ }^{*}$ Address correspondence to: Devon E. Romero, University of Texas at San Antonio, Department of Counseling, 501 W. Cesar E. Chavez, San Antonio, TX 78207, USA. Email: devon.romero@utsa.edu

Copyright: (c) 2020. Romero et al. This is an Open Access article distributed under the terms of the Creative Commons Attribution License (CC-BY).

\section{Edited by:}

Rex L. Cannon, PhD, SPESA Research Institute, Knoxville, Tennessee, USA

\section{Reviewed by:}

Rex L. Cannon, PhD, SPESA Research Institute, Knoxville, Tennessee, USA

Randall Lyle, PhD, Mount Mercy University, Cedar Rapids, lowa, USA

\section{Introduction}

According to the National Center for posttraumatic stress disorder (PTSD), around $8 \%$ of the population will experience PTSD symptoms at some point in their lives. PTSD used to be associated mainly with veterans and refugees, but we now know that PTSD can occur after any witnessed or experienced upsetting traumatic event. The experience of a traumatic event can also lead to maladaptive outcomes in social behaviors, physiology, emotions, and cognitions including affect dysregulation. PTSD can develop in nearly one in eight adult trauma survivors (Jones, Rybak, \& Russell-Chapin, 2017). Exposure to a traumatic event may lead to maladaptive stress responses. These responses serve as signifiers of how the body remembers and re-experiences stressors when triggered (Othmer \& Othmer, 2009). These stress responses include but are not limited to over arousal, hypervigilance, flash backs, nightmares, and fear. Survivors of trauma may also have difficulty with affective prosody and lack the ability to properly interpret emotional cues of language (Jones et al., 2017).

There are various therapeutic approaches to treating PTSD and other related trauma symptoms. Previous forms of treatment include prolonged exposure therapy which is an integration of imaginal exposure and in vivo exposure (McLean \& Foa, 2013). The clinician exposes the client and grounds them in real time to teach coping skills. This is a form of cognitive behavior therapy (CBT) and similar to cognitive processing therapy (CPT), another popular treatment for PTSD. CPT aims to reframe maladaptive thinking and build new skills to identify and address these maladaptive thoughts (Schumm, Dickstein, Walter, Owens, \& Chard, 2015). An additional form of treatment that addresses maladaptive thoughts is eye movement desensitization and reprocessing (EMDR). EMDR desensitizes traumatic memories by having the client retell the event while watching the clinician's rapid finger movements (National Library of Medicine, 2002). The common theme amongst PTSD treatment is an attention to the thoughts and memories 
associated with the event. Although there are various approaches that target these thoughts and memories once told aloud to the clinician, the goal of neurofeedback is to create better function in the affected areas of the brain through brainwave training (Othmer \& Othmer, 2009).

Neurofeedback, formerly known as electroencephalography (EEG) biofeedback, is a form of biofeedback that uses real-time displays of neural activity to teach the client self-regulation of brain function. Neurofeedback protocols supply participants with audio or visual feedback, or both, which trains them to maintain certain frequency bands (Marzbani, Marateb, \& Mansourian, 2016). From this viewpoint, mental health concerns present as variations in brain wave frequencies. This modality improves health, performance, and the physiological changes which often occur in conjunction with changes to thoughts, emotions, and behavior. Neurofeedback uses precise protocols for the purposes of optimizing brain wave activity, done subconsciously through a loop process, using operant conditioning (Sitaram et al., 2016). Reports of neurofeedback as an effective treatment for PTSD date back to the original study by Peniston and Kulkosky (1991) on alpha-theta neurofeedback for Vietnam veterans. A pilot study on neurofeedback for chronic PTSD by Gapen et al. (2016) showed promising results, with a follow-up randomized, waitlist-controlled study demonstrating statistically robust results (van der Kolk et al., 2016).

Using a waitlist control, van der Kolk et al. (2016) examined the effect of neurofeedback treatment for those who met the criteria for chronic PTSD. Participants in the experimental group received neurofeedback training twice a week for up to 30 minutes for a total of 24 sessions. Training focused on decreasing 2-6 Hz and 22-36 Hz while increasing $10-13 \mathrm{~Hz}$ with a placement of $\mathrm{T} 4$ as the active site and P4 as the reference site. The authors found those who received neurofeedback training had significant improvements such as improvement of PTSD symptomatology, affect regulation, tension reduction activities, identity impairment, and abandonment concerns (van der Kolk et al., 2016). van der Kolk's (2016) underlying theme of "rewiring" the brain as a necessary proponent of change supports the argument that traditional therapeutic methods do not suffice in treating PTSD. Interventions such as neurofeedback can change the way in which we treat mental health disorders as well as how we conceptualize them. The intent of this study is to provide further evidence that neurofeedback provides significant care through neuronal regulation and stabilization.

\section{Purpose of Study}

This study examined the effectiveness of neurofeedback training for individuals presenting with a primary concern of PTSD symptoms. The overarching goal was to evaluate the effectiveness of neurofeedback training on lowering PTSD symptoms using the same protocol and symptom scales as van der Kolk et al. (2016). Therefore, we examined the following research questions:

1. Is there a statistically significant difference on participant self-reported PTSD symptom severity and frequency as measured by the Davidson Trauma Scale following neurofeedback training?

2. Is there a statistically significant difference on participant self-reported emotion regulations and interpersonal processes as measured by the Inventory of Altered Self-Capacities following neurofeedback training?

We anticipated improvement in PTSD symptom severity and frequency and affect regulation. We based our hypotheses on the literature and statistically significant findings of van der Kolk et al. (2016).

\section{Method}

This within-subjects research design occurred in a counseling center in a university counseling department with the primary purpose of training graduate-level counseling students. The present study evaluated the effectiveness of neurofeedback to increase affect regulation and reduce PTSD related symptoms in adults.

\section{Participants}

The present study included data from a total of 21 clients ranging in age from 18 to $68(M=40.86, S D=$ $14.32)$ with $38 \%(n=8)$ of clients self-reporting as male and $62 \%(n=13)$ self-reporting as female (see Table 1). Clients from the surrounding community seeking neurofeedback services for PTSD and trauma-related symptoms contacted the counseling department clinic at a southern United States university. This counseling center was well situated geographically and demographically and was one of the few clinics in its community providing free mental health services to the general public. The counseling department clinic has a history of providing services free of charge to the surrounding community. Thus, 
the primary method of recruitment included referrals from practitioners in the community. Inclusion criteria consisted of primary trauma symptoms, availability, and age requirements. Clients agreed to attend a minimum total number of 15 neurofeedback training sessions, twice per week. Clients received neurofeedback services free of charge.

\begin{tabular}{|c|c|c|c|}
\hline \multicolumn{4}{|c|}{$\begin{array}{l}\text { Table } 1 \\
\text { Client Demographics }\end{array}$} \\
\hline Client \# & Age & Gender & $\begin{array}{l}\text { Number of } \\
\text { Sessions }\end{array}$ \\
\hline 1 & 30 & $\mathrm{~F}$ & 15 \\
\hline 2 & 37 & $M$ & 11 \\
\hline 3 & 18 & $F$ & 18 \\
\hline 4 & 46 & $M$ & 18 \\
\hline 5 & 45 & $F$ & 16 \\
\hline 6 & 18 & $F$ & 13 \\
\hline 7 & 54 & $M$ & 9 \\
\hline 8 & 43 & $F$ & 21 \\
\hline 9 & 33 & $\mathrm{~F}$ & 16 \\
\hline 10 & 48 & $F$ & 15 \\
\hline 11 & 41 & $M$ & 11 \\
\hline 12 & 47 & $F$ & 17 \\
\hline 13 & 49 & $\mathrm{~F}$ & 17 \\
\hline 14 & 67 & $F$ & 13 \\
\hline 15 & 68 & $M$ & 13 \\
\hline 16 & 40 & $M$ & 19 \\
\hline 17 & 27 & $\mathrm{~F}$ & 14 \\
\hline 18 & 18 & $M$ & 18 \\
\hline 19 & 56 & $\mathrm{~F}$ & 19 \\
\hline 20 & 30 & $F$ & 15 \\
\hline 21 & 30 & $M$ & 17 \\
\hline$M(S D)$ & $40.86(14.32)$ & -- & $15.48(3.04)$ \\
\hline
\end{tabular}

\section{Clinicians}

Student and volunteer clinicians provided neurofeedback services. Student clinicians consisted of clinical mental health master-level students, school counseling master-level students, and counselor education and supervision doctoral-level students within counseling programs nationally accredited by the Council for Accreditation of Counseling and Related Education Programs (CACREP). Volunteer clinicians consisted of faculty and/or credentialed local clinicians such as licensed professional counselors, psychologists, neuropsychologists, nurse practitioners, and social workers. All clinicians (i.e., volunteer, student) previously completed the Biofeedback Certification International Alliance (BCIA) requirements for didactic coursework for neurofeedback and are under the supervision of a certified and licensed supervisor. Regarding the completed didactic coursework, all student clinicians completed an introduction to neurofeedback course offered in their program of study that is based on the certification requirements of $\mathrm{BCIA}$. At the time of data collection, student clinicians enrolled in advanced neurofeedback or practicum of neurofeedback courses provided the neurofeedback services to the study participants.

\section{Measures}

Davidson Trauma Scale. The Davidson Trauma Scale (DTS; Davidson, 1996) is a 17-item self-report measure that assesses PTSD symptoms as defined in the Diagnostic and Statistical Manual of Mental Disorders (DSM-IV; Davidson, 1996). The DTS has three symptoms clusters: Intrusion, Avoidance/ Numbing, and Hyperarousal with scores ranging from 0 to 136 (Davidson, 1996). Measures of Severity and Frequency of symptoms occur on a 5 -point scale $(0=$ Not at all to $4=$ Every day). Example items include "Have you been upset by something that reminded you of the event?" and "Have you felt distant or cut off from other people?" (Davidson, 1996). The DTS shows good reliability with a high Cronbach's alpha of over .90 for the entire scale as well as the Frequency and Severity scales (Davidson, 1996). The DTS demonstrated a preintervention Cronbach's alpha of .79 with two removed cases due to missing item level data and a postintervention Cronbach's alpha of .95 for the current sample.

Inventory of Altered Self-Capacities. The Inventory of Altered Self-Capacities (IASC; Briere, 2000 ) is a 63-item self-report. The IASC consists of seven scales that assess self-related psychological difficulties using the following domains: Interpersonal Conflicts, Idealization-Disillusionment, Abandonment Concerns, Identity Impairment, Susceptibility to Influence, Affect Dysregulation, and Tension Reduction Activities. Of those scales, Identity Impairment and Affect Dysregulation each have two subscales. Self-awareness and Identity Diffusion comprise the Identity Impairment scale and Affect Instability and Affect Skills Deficits are the two subscales within the Affect Dysregulation scale. 
Ratings of items occur according to frequency of occurrence over the last six months on a 5-point Likert-type scale $(1=$ Never to $5=$ Very often $)$. Example items include "Doing things to stop feeling so much pressure or pain inside," "Suddenly hating someone you used to like a lot," and "Wishing you could calm down but not being able to" (Briere \& Runtz, 2002). The IASC shows good reliability with the Cronbach's alpha ranging from .78 to .93 across its scales and subscales (Briere \& Runtz, 2002). In this sample, the IASC demonstrated a preintervention Cronbach's alpha of .97 and a postintervention Cronbach's alpha of .96 with two removed cases due to missing item-level data.

Data Collection Procedures. The Institutional Review Board at a southern United States university approved this study. During the preintervention phase, clients completed the informed consent process and both outcome-based measures (i.e., DTS, IASC). Clients also completed both outcomebased measures postintervention. This section describes procedures for the neurofeedback training process.

Clinicians utilized BrainMaster Atlantis two-channel amplifiers (BrainMaster Technologies, Inc., Bedford, $\mathrm{OH}$ ) and BioExplorer software (CyberEvolution, Inc., Seattle, WA) for neurofeedback training. Clinicians cleaned the neurofeedback sites with rubbing alcohol and PCl prep pads. Next, clinicians applied Ten20 EEG conductive paste to gold-plated electrodes and placed them on a client's scalp. Throughout the neurofeedback training process, clinicians noted the impedance levels and adjusted as needed to ensure a reading of less than five $\mathrm{k} \Omega$ (Jones, 2015).

The range of attended sessions was 9-21 $(M=15.48$, $S D=3.04$ ) for approximately 20 minutes per session, twice per week, over the course of one academic semester. The neurofeedback clinicians asked clients to halt the consumption of caffeine or other potential nonessential substances on neurofeedback training days. During the training sessions, protocols consisted of amplitude uptraining and/or downtraining preselected frequency bands consistent with van der Kolk et al. (2016). The thresholds are set at the beginning of each session with an ideal reward rate of $50 \%$. Following the procedures of van der Kolk et al. (2016), training sites for all clients include T4 as the active site, $\mathrm{P} 4$ as the reference site, and $\mathrm{A} 1$ as the ground. Further, the aim for neurofeedback training is for clients to decrease brain activity of 2-6 $\mathrm{Hz}$ and 22-36 Hz and increase 10-13 Hz (van der Kolk et al., 2016). Due to the varying degree of counseling skills present among the student clinicians and volunteers, clinicians received instructions to limit counseling interventions to those necessary for neurofeedback training, such as shaping behavior, positive reinforcement, and emotional support.

Statistical Analysis. We used the Statistical Package for the Social Sciences (SPSS) software version 25 (SPSS, 2017) for all statistical analyses. Before analysis, we examined cases for missing data, outliers, and normality. The percentage of participants missing data ranged from $0 \%$ for the DTS to $.90 \%$ for the IASC. Following Cohen's (1988) conventions, a medium effect size of .5, error probability of .05 , and 21 participants, A post-hoc G*Power analysis determined that the current sample size of this study would yield a power of .59 .

\section{Results}

We used a paired samples $t$-test to measure pre-post comparisons of the DTS symptom clusters, Severity, Frequency, and Total scores which resulted in statistically significant improvements. Table 2 presents a summary of these results. On the Total DTS score, for all subjects, the mean of the prescores was $78.10(S D=25.02)$ and the mean of the postscores was $61.52(S D=35.38)$. The paired samples $t$-test yielded a statistically significant improvement, with $t(20)=2.95, p=.008$, with a medium effect size $(d=.64)$. See Table 3 for the prepost DTS scores for each client.

On the IASC, six of the seven scales demonstrated statistically significant improvement. In addition, we found significant mean differences for Identity Diffusion, one of two Identity Impairment subscales, and both of the Affect Dysregulation subscales (i.e., Affect Skills Deficits, Affect Instability). We present a summary of these results in Table 4.

\section{Discussion}

This study builds on the findings of van der Kolk et al. (2016) by examining the effectiveness of neurofeedback training for individuals presenting to a counseling center in a university counseling department with a primary concern of PTSD symptoms. The overarching goal was to evaluate the effectiveness of neurofeedback training to lower PTSD symptoms using an existing protocol previously evaluated in a randomized, waitlistcontrolled efficacy trial. van der Kolk et al. (2016) found 24 sessions of neurofeedback resulted in significant improvements in PTSD symptomology and 


\begin{tabular}{lccccc}
\hline $\begin{array}{l}\text { Table } 2 \\
\text { Davidson Trauma Scale }\end{array}$ & & & & & \\
\hline \multicolumn{1}{l}{ Pre $M(S D)$} & Post $M(S D)$ & $t(d f)$ & $p$ & $d$ \\
\hline Intrusion & $21.38(9.98)$ & $17.57(11.14)$ & $1.94(20)$ & .067 & .42 \\
Avoidance/Numbing & $30.81(11.02)$ & $24.57(14.82)$ & $2.20(20)$ & .040 & .48 \\
Hyperarousal & $25.90(9.14)$ & $19.48(11.83)$ & $3.44(20)$ & .003 & .75 \\
Severity & $38.67(14.98)$ & $30.90(17.99)$ & $2.41(20)$ & .025 & .53 \\
Frequency & $39.43(11.57)$ & $30.71(18.20)$ & $3.39(20)$ & .003 & .74 \\
Total & $78.10(25.02)$ & $61.52(35.38)$ & $2.95(20)$ & .008 & .64 \\
\hline
\end{tabular}

\section{Table 3}

Davidson Trauma Scale Total Scores

\begin{tabular}{|c|c|c|}
\hline Client \# & Pre & Post \\
\hline 1 & 87 & 72 \\
\hline 2 & 113 & 114 \\
\hline 3 & 41 & 14 \\
\hline 4 & 96 & 127 \\
\hline 5 & 50 & 31 \\
\hline 6 & 79 & 86 \\
\hline 7 & 58 & 22 \\
\hline 8 & 63 & 72 \\
\hline 9 & 103 & 73 \\
\hline 10 & 58 & 19 \\
\hline 11 & 96 & 69 \\
\hline 12 & 93 & 108 \\
\hline 13 & 121 & 105 \\
\hline 14 & 92 & 61 \\
\hline 15 & 105 & 40 \\
\hline 16 & 88 & 66 \\
\hline 17 & 75 & 92 \\
\hline 18 & 34 & 55 \\
\hline 19 & 38 & 11 \\
\hline 20 & 68 & 22 \\
\hline 21 & 82 & 33 \\
\hline$M(S D)$ & $78.10(25.02)$ & $61.52(35.38)$ \\
\hline
\end{tabular}

areas of psychological functioning capacity (i.e., affect regulation, tension reduction activities, identity impairment, abandonment concerns) using T4 as the active site and P4 as the reference site. The average session attendance total for the present study was 15 sessions. This study produced similar, promising results. Findings revealed statistically significant improvement in avoidance/numbing, hyperarousal, severity, frequency, and overall total score for the DTS. For instance, the Cohen's effect size value for the DTS overall total score $(d=.64)$ suggested a moderate practical significance, whereas van der Kolk and colleagues (2016) found a large effect size $(d=.92)$ difference between pre- and postassessment.

Similar to van der Kolk et al.'s (2016) findings, neurofeedback participants in the present study demonstrated statistically significant improvements in affect regulation as the aim of neurofeedback is neuronal regulation and stabilization. More specifically, the present study resulted in statistically significant improvements in measures of interpersonal conflicts, idealization-disillusionment, abandonment concerns, identity impairment, susceptibility to influence, affect dysregulation, and tension reduction for the IASC. As shown in Table 4, effect sizes for these measures demonstrated a range of small to large practical significance.

In addition, van der Kolk et al. (2016) expressed how neurofeedback can be a promising change agent for habitual dysfunctional neuronal patterns and highlighted its potential of becoming widely available in community settings as it is economically accessible and it does not have to be administered out of a research clinic such as the present study. Although

Note. $t(20)=2.95, p=.008$ 


\begin{tabular}{lrrrrc}
\hline $\begin{array}{l}\text { Table } 4 \\
\text { Inventory of Altered Self-Capacities }\end{array}$ & & & & & \\
\hline & Pre $M(S D)$ & Post $M(S D)$ & $t(d f)$ & \multicolumn{1}{c}{} & \multicolumn{1}{c}{. } \\
\hline Interpersonal Conflicts & $23.42(7.81)$ & $20.47(8.17)$ & $2.40(18)$ & .027 & .55 \\
Idealization-Disillusionment & $19.21(6.07)$ & $16.00(3.46)$ & $2.54(18)$ & .021 & .58 \\
Abandonment Concerns & $23.05(9.17)$ & $18.58(8.78)$ & $3.41(18)$ & .003 & .78 \\
Identity Impairment & $26.63(9.15)$ & $22.37(8.65)$ & $2.28(18)$ & .035 & .52 \\
Self-Awareness & $16.32(5.58)$ & $14.47(5.98)$ & $1.40(18)$ & .178 & .32 \\
Identity Diffusion & $9.89(4.69)$ & $7.89(3.64)$ & $2.41(18)$ & .027 & .55 \\
Susceptibility to Influence & $19.32(7.92)$ & $15.00(5.56)$ & $3.12(18)$ & .006 & .72 \\
Affect Dysregulation & $26.79(8.22)$ & $22.79(8.78)$ & $2.91(18)$ & .009 & .67 \\
Affect Skill Deficits & $14.32(5.53)$ & $12.32(5.19)$ & $2.39(18)$ & .028 & .55 \\
Affect Instability & $12.47(3.42)$ & $9.95(3.49)$ & $3.66(18)$ & .002 & .84 \\
Tension Reduction & $18.42(5.94)$ & $15.79(5.46)$ & $1.99(18)$ & .062 & .46 \\
\hline
\end{tabular}

neurofeedback is more affordable than other neuroimaging methods, the cost of training, equipment, software, and supplies (e.g., electrodes, conductive paste) is a significant barrier for settings with limited resources and funding (Beeson \& Field, 2017).

Furthermore, for the present study, both student and volunteer clinicians provided neurofeedback services. Student clinicians included those enrolled in an accredited counseling graduate degree program and volunteer clinicians included both community members and faculty from the study's institution. While student and volunteer clinicians completed their didactic coursework, they were still engaging in components of their mentoring and practical skills training and had not yet completed their neurofeedback certification exam. There were benefits to incorporating volunteer clinicians, some of which are local mental health professionals in the community. This had a positive impact of creating networks with student clinicians, as well as providing "real world" perspectives of the local mental health community. However, while this created a greater level of feasibility and accessibility to community engagement and pursuing neurofeedback research, there were also limitations to having variance in training and skill level, which the authors discuss further in the limitations section.
Limitations and Implications for Future Research There are important limitations in the present research to consider prior to the interpretation of findings. Concerning the demographics of the participants, researchers only collected gender and age preventing the researchers from analyzing the data beyond the current scope and geographically. The study also lacked a control or sham group and had a fairly small sample size which limits the power but also risks Type II errors and generating biased interpretations. Furthering this, the psychometrics of the scales should need further examination as they are at least two decades old and may not be relevant to the participants in this research, ultimately impacting the reliability, validity, and fairness. Methodological limitations include the within-subjects design with no control group. Also, in relation to the number of sessions attended by each individual, client participation in neurofeedback training varied. Not all clients attended the agreed upon 15 sessions. As such, the researchers set a session cut-off of nine sessions for inclusion in the present study. Removal of other client data not meeting this criterion accounts for the limited sample size and resulting statistical interpretation. Thus, replication of this study may have alternative results considering the sample size, demographics, and psychometric relevance.

There are many implications of this study in relation to future research directions and approaches to neurofeedback in the mental health field. This 
present research and van der Kolk et al.'s (2016) study focused on T4 and P4 locations, however multicultural and individual differences could impact how this data could be interpreted. While there are emerging neuro-informed frameworks regarding mental health disorders, suggesting abnormalities in neural connectivity and brain wave patterns (van der Kolk et al., 2016), the present researchers strongly suggest contextualizing individuals by understanding their sociocultural and social justice implications. Therefore, the present researchers encourage promoting individual protocols and within-subject designs but not comparisons between participants and across populations and genders.

There is a colonizing to mental health and a lack of cultural diversity in the literature that researchers should acknowledge as these factors impact neurofeedback data and results could deviate from the image of a "normal" brain and regular levels of stress. Given that there are biological mechanisms of a life of marginality (Douthit \& Russotti, 2017), researchers have an ethical responsibility to acknowledge the social justice implications of their research. In regard to future studies, a neuroecological approach (Sherry, 2006; van Dijk \& Myin, 2019) could create a more ethical and social justice-oriented paradigm to investigate similar research.

Researchers should also consider epigenetics and psychoneuroimmunology, or $\mathrm{PNI}$, in how this relates to all of neuroscience, neurofeedback research, and understanding the mind-brain connection. In specific regards to research relating to anxiety, stress, and PTSD, epigenetic modifications of the hypothalamic pituitary adrenal (HPA) axis gene can create more reactivity to stress and a predispositional vulnerability to psychological manifestations of chronic stress (Douthit \& Russotti, 2017). Whereas PNI has the role of translating chronic environmental stress into physiological susceptibility in individuals, these factors can impact the immune system and encourage inflammation, creating a stress-immune dysregulation relationship that relates to mind-body communication which affects overall mental health and predisposed risks or vulnerabilities to future stress (Douthit \& Russotti, 2017).

Future research should consider how to advise counselors and counselor educators who intend to use neurofeedback in education and practice as well as conceptualize how to standardize training and access to equipment. This study did not investigate the combination of talk therapy or counseling with neurofeedback; future research should further investigate the partnering of both. Finally, future researchers should also consider using qualitative methods to gain an understanding of participant experiences with neurofeedback training used for the treatment of PTSD and trauma-related symptoms.

\section{Conclusion}

This study explored specific neurofeedback protocols for trauma treatment. The results supported our hypotheses that neurofeedback would significantly improve PTSD symptom severity and frequency and affect regulation. Although this study contains limitations, the results are promising for using neurofeedback to treat trauma. Neurofeedback can better inform interventions and provide a physical representation of mental manifestations as well their relationship to emotions, physiology, and social justice (Douthit \& Russotti, 2017). Future mental health models should account for emerging mindbody paradigms but also understand the social justice implications associated with them. Future research is essential and can begin with the implications for research presented in this article.

\section{Author Declarations}

The authors have no financial interests, grant support, or conflicts of interest to report.

\section{References}

Beeson, E. T., \& Field, T. A. (2017). Conducting brain-based research and program evaluation. In T. A. Field, L. K. Jones, \& L. A. Russell-Chapin (Eds.), Neurocounseling: Brain-based clinical approaches (pp. 213-226). Alexandria, VA: American Counseling Association. https://doi.org/10.1002 19781119375487.ch13

Briere, J. (2000). Inventory of Altered Self-Capacities professional manual. Odessa, FL: Psychological Assessment Resources.

Briere, J., \& Runtz, M. (2002). The Inventory of Altered SelfCapacities (IASC): A standardized measure of identity, affect regulation, and relationship disturbance. Assessment, 9(3), 230-239. https://doi.org/10.1177/1073191102009003002

Cohen, J. (1988). Statistical power analysis for the behavioral sciences (Rev. ed.). New York, NY: Academic Press.

Davidson, J. (1996). Davidson trauma scale professional manual. North Tonawanda, NY: Multi-Health Systems Inc.

Douthit, K. Z., \& Russotti, J. (2017). Biology of marginality: A neurophysiological exploration of social and cultural foundations for psychological health. In T. A. Field, L. K. Jones, \& L. A. Russell-Chapin (Eds.), Neurocounseling: Brain-based clinical approaches (pp. 45-60). Alexandria, VA: American Counseling Association. https://doi.org/10.1002 19781119375487.ch3

Gapen, M., van der Kolk, B. A., Hamlin, E., Hirshberg, L., Suvak, M., \& Spinazzola, J. (2016). A pilot study of neurofeedback for chronic PTSD. Applied Psychophysiology and Biofeedback, 41(3), 251-261. https://doi.org/10.1007/s10484-015-9326-5

Jones, M. S. (2015). Comparing DC offset and impedance readings in the assessment of electrode connection quality. NeuroRegulation, 2(1), 29-36. https://doi.org/10.15540 /nr.2.1.29 
Jones, L. K., Rybak, C., \& Russell-Chapin, L. A. (2017). Neurophysiology of traumatic stress. In T. A. Field, L. K. Jones, \& L. A. Russell-Chapin (Eds.), Neurocounseling: Brain-based clinical approaches (pp. 61-80). Alexandria, VA: American Counseling Association. https://doi.org/10.1002 /9781119375487.ch4

Marzbani, H., Marateb, H. R., \& Mansourian, M. (2016). Neurofeedback: A comprehensive review on system design, methodology and clinical applications. Basic and Clinical Neuroscience, $\quad 7(2), \quad$ 143-158. https://doi.org/10.15412 /J.BCN.03070208

McLean, C. P., \& Foa, E. B. (2013). Dissemination and implementation of prolonged exposure therapy for posttraumatic stress disorder. Journal of Anxiety Disorders, 27(8), 788-792. https://doi.org/10.1016/j.janxdis.2013.03.004

National Library of Medicine. (2002). EMDR. The Harvard Mental Health Letter, 18(8), 4-5. Retrieved from http://search.proquest.com/docview/71492232/

Othmer, S., \& Othmer, S. F. (2009). Post traumatic stress disorder-The neurofeedback remedy. Biofeedback, 37(1), 24-31. Retrieved from http://search.proquest.com/docview /208152800/

Peniston, E. G., \& Kulkosky, P. J. (1991). Alpha-theta brainwave neuro-feedback for Vietnam veterans with combat-related post-traumatic stress disorder. Medical Psychotherapy, 4, 4760.

Schumm, J. A., Dickstein, B. D., Walter, K. H., Owens, G. P., \& Chard, K. M. (2015). Changes in posttraumatic cognitions predict changes in posttraumatic stress disorder symptoms during cognitive processing therapy. Journal of Consulting and Clinical Psychology, 83(6), 1161-1166. https://doi.org/10.1037/ccp0000040

Sherry, D. F. (2006). Neuroecology. Annual Review of Psychology, 57, 167-197. https://doi.org/10.1146 /annurev.psych.56.091103.070324

Sitaram, R., Ros, T., Stoeckel, L., Haller, S., Scharnowski, F. Lewis-Peacock, J., ... Sulzer, J. (2016). Closed-loop brain training: The science of neurofeedback. Nature Reviews Neuroscience, $\quad 18(2), \quad 86-100 . \quad$ https://doi.org/10.1038 /nrn.2016.164

van der Kolk, B. A., Hodgdon, H., Gapen, M., Musicaro, R., Suvak, M. K., Hamlin, E., \& Spinazzola, J. (2016). A randomized controlled study of neurofeedback for chronic PTSD. PLOS ONE, 11(12), e0166752. https://doi.org/10.1371 /journal.pone.0166752

van Dijk, L., \& Myin, E. (2019). Ecological neuroscience: From reduction to proliferation of our resources. Ecological Psychology: Gibsonian Neuroscience, 31(3), 254-268. https://doi.org/10.1080/10407413.2019.1615221

Received: June 30, 2020

Accepted: August 31, 2020

Published: September 30, 2020 